\title{
In Vivo Effects of Protease Inhibitors on Chickens with Hereditary Muscular Dystrophy
}

\author{
Michael S. Hudecki, Catherine M. Pollina, and Reid R. Heffner, Departments \\ of Biological Sciences and Pathology, State University of New York at Buffalo, \\ New York 14260
}

A B S T R A C T Beginning on day 4 ex ovo, and every 3 $\mathrm{d}$ thereafter, genetically dystrophic Line 413 chickens were given intraperitoneal injections ( $4 \mathrm{mg} / \mathrm{kg}$ body wt) of a protease inhibitor, leupeptin, pepstatin, or antipain. Experimental chickens received protease inhibitors dissolved in a water:ethanol:dimethyl sulfoxide solution (50:40:10, vol:vol:vol). Control untreated animals received diluent injections.

Untreated dystrophic chickens typically reach around day 30 ex ovo a maximum ability to right from the supine position in a standardized functional test for muscle weakness. After day 30 ex ovo, the dystrophic chickens are found to decline progressively in their ability to right, compared with normal, nondystrophic controls, which have an unimpaired ability to right. Concomitantly, dystrophic chickens exhibit characteristically high levels of plasma creatine phosphokinase. enzyme activity. In addition, an increased frequency of degenerating, regenerating, and vacuolated myofibers, and inflammatory cells appear in the affected pectoralis major muscles from the dystrophic chicken.

Throughout the duration of the trial, there was no major enhancement in the functional righting ability of dystrophic chickens receiving any one of the protease inhibitors tested. However, there was a significant reduction in the abnormally high levels of plasma creatine phosphokinase in the treated chickens. Also, there was an apparent reduction in the mean number of vacuolated fibers in the pectoralis muscle from the protease inhibitor-treated birds. No significant reductions were observed in the relative frequency of degenerating and regenerating myofibers or inflammatory cells. In addition to the plasma creatine phosphokinase decrease, however, therapeutic benefit was seen in $31.0,30.5$, and $14.8 \%$ increases in the wet weight (and total noncollagen protein) of pectoralis muscle from

Received for publication 25 August 1980 and in revised form 2 December 1980. dystrophic chickens receiving leupeptin, pepstatin or antipain, respectively.

\section{INTRODUCTION}

Substantial elevation in proteolytic enzyme activity is associated with the progressive loss in muscle tissue exhibited in both genetically dystrophic humans (1-3) and animals (4-7). Since the pioneering biochemical studies of Umezawa and coworkers $(8,9)$, in which microbial inhibitors to proteolytic activity were isolated, evidence has indicated that some of these inhibitors (e.g., leupeptin, pepstatin, and antipain) may have a therapeutic role in retarding the abnormal muscle protein degradation found in hereditary myopathies. In this regard, the addition of protease inhibitors in vitro to embryonic, intact, or homogenate muscle preparations from dystrophic animals promotes increased protein synthesis (10), decreased protein breakdown (10-13), and decreased atrophy in general (14).

There has been a lack of trials in vivo involving protease inhibitor treatment of animals with genetic myopathies. Results to date are inconclusive; for example, when dystrophic mice were given either subcutaneous or intraperitoneal dosages of protease inhibitors, benefit was seen in one study (15), but not in another (16). In another report (17), improvements in muscle mass and histology and significant reductions in abnormally high levels of serum creatine phosphokinase activity $(\mathrm{CPK})^{1}$ were found in genetically dystrophic chickens injected with leupeptin and pepstatin intramuscularly.

The purpose of the present study was to investigate further the effects in vivo of individual protease inhibitors on the genetically dystrophic Line 413 chicken within an established, standardized, drug screening program (18-21). Reported here is new evidence for limited degrees of drug-related improvements in a

\footnotetext{
${ }^{1}$ Abbreviations used in this paper: CPK, creatine phosphokinase activity; FN, flip number.
} 
functional test for righting ability and plasma CPK levels, and in muscle mass and histology.

\section{METHODS}

Line 413 dystrophic and genetically related Line 412 normal, nondystrophic chickens were purchased as eggs from the Department of Avian Sciences, University of California, Davis. The incubation conditions and subsequent care and maintenance of the mostly female chicks were as previously described (18-21).

Starting on day 4 ex ovo, each experimental chicken received an intraperitoneal injection of $0.5 \mathrm{ml}$ protease inhibitor solution ( $4 \mathrm{mg} / \mathrm{kg}$ body wt) every $3 \mathrm{~d}$ between 0800-1000 h. Each protease inhibitor was dissolved in a water:ethanol:dimethylsulfoxide solution (50:40:10, vol:vol: vol). A number of dystrophic chickens in each trial received diluent only and served as untreated dystrophic controls. All animals were weighed twice a week.

The functional assessment of muscle strength was measured with the extensively described flip test procedure (18-21). In brief, each drug-treated and untreated control chicken was given a standardized test for righting from the supine position every $5 \mathrm{~d}$ between 1300 and $1500 \mathrm{~h}$. The number of successful attempts to regain an upright position scored out of five consecutive opportunities was defined as the flip number (FN). To eliminate bias in testing, all chickens were pooled before the testing procedure. Subsequently, each bird was randomly selected for the righting test, and the $\mathrm{FN}$ later matched with its wing band number.

Blood for the determination of plasma CPK was obtained without anesthesia from the jugular vein into heparinized syringes weekly between 1000 and $1200 \mathrm{~h}$. Plasma was separated by centrifugation at $755 \mathrm{~g}$ for $15 \mathrm{~min}$ at $4^{\circ} \mathrm{C}$. On the same day on coded samples, plasma CPK activities were assayed by an ultraviolet spectrophotometric procedure, using the activated CPK reagent set of Boehringer Mannheim Biochemicals, Indianapolis, Ind. (cat. No. 124176). Enzyme activity was expressed in International milliunits CPK per milliliter plasma at $25^{\circ} \mathrm{C}, \mathrm{pH}$ 7.0. Plasma dilutions were made when enzyme activities were near or above the linear range of the assay conditions.

To test the possible inhibitory effect of the protease inhibitors directly on CPK, aliquots of plasma with known CPK activity were assayed individually with the addition of 0.081 $\mathrm{mM}$ leupeptin, $0.054 \mathrm{mM}$ pepstatin, or $0.062 \mathrm{mM}$ antipain. The water:ethanol:dimethyl sulfoxide diluent (50:40:10, vol:vol:vol) of the protease inhibitors was also tested at the same final concentration of $1.8 \%$ for its effect on the assay of CPK. As a result, neither the protease inhibitors nor the diluent was found to have an effect on the quantitation in vitro of plasma CPK activities.

In the histological survey of muscle involved in the righting reflex, samples of pectoralis major muscle from each chicken were removed at day 67 ex ovo after completion of the drug trials. As previously described (20), each muscle sample was excised, weighed, and placed in an isometric clamp before immersion in $4 \%$ paraformaldehyde, fixed for $18-24 \mathrm{~h}$, and embedded in paraffin. 4-6- $\mu \mathrm{m}$ sections were stained with hematoxylin, eosin, and Masson's trichrome. Four pathologic criteria were routinely examined and quantitated in muscle specimens from each bird: vacuolation, fiber necrosis, inflammation, and fiber regeneration. Employing a calibrated grid, 16 individual microscopic fields per muscle were examined and each of the above pathologic features was enumerated. The frequencies of necrotic and regenerative fibers were grouped together. Values were expressed as the mean number of observations per square millimeter.

Total noncollagen protein of the pectoralis major muscle from the experimental chickens was determined in homogenates by the procedure of Lowry et al. (22), using bovine serum albumin as standard.

Leupeptin, pepstatin, and antipain were donated by Dr. Walter Troll, New York University School of Medicine, New York, Dr. Arthur M. Dannenberg, Department of Environmental Medicine, Johns Hopkins University, Baltimore, Md., and Dr. Hamao Umezawa, Institute of Microbial Chemistry, University of Tokyo, Japan. All chemicals were reagent grade. Statistical tests of significance of paired means were derived from the two-sided Students' $t$ test.

\section{RESULTS}

Flip test for righting ability. Dystrophic chickens treated with leupeptin exhibited a temporary yet significant $(P<0.001)$ prolongation in a functional test for muscle strength (Fig. 1). As seen here, and has been reported extensively elsewhere (19-21), the untreated dystrophic control Line 413 chickens reached an optimal FN around day 30 ex ovo and subsequently declined. However, leupeptin-treated chickens maintained a perfect $\mathrm{FN}$ of five for 2 additional wk before decreasing in righting ability.

There were no significant differences evident in the righting abilities of dystrophic Line 413 chickens treated with either pepstatin (Fig. 2) or antipain (Fig.

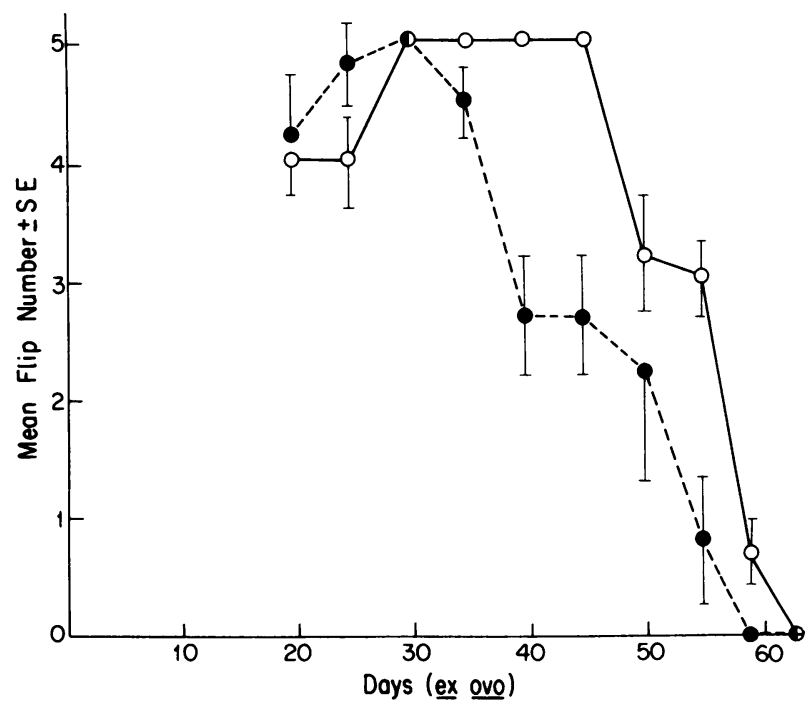

FIGURE 1 Effect of leupeptin on mean FN of Line 413 dystrophic chickens. The mean FN of the leupeptin-treated group $(O)$ at days 39 and 44 ex ovo are significantly different from the corresponding untreated control group $(0)$ at $P$ $<0.001$. Details of dosage schedule and procedure for flip testing are as described in Methods. The number of animals per point is four untreated dystrophic control and six leupeptin-treated dystrophic. 


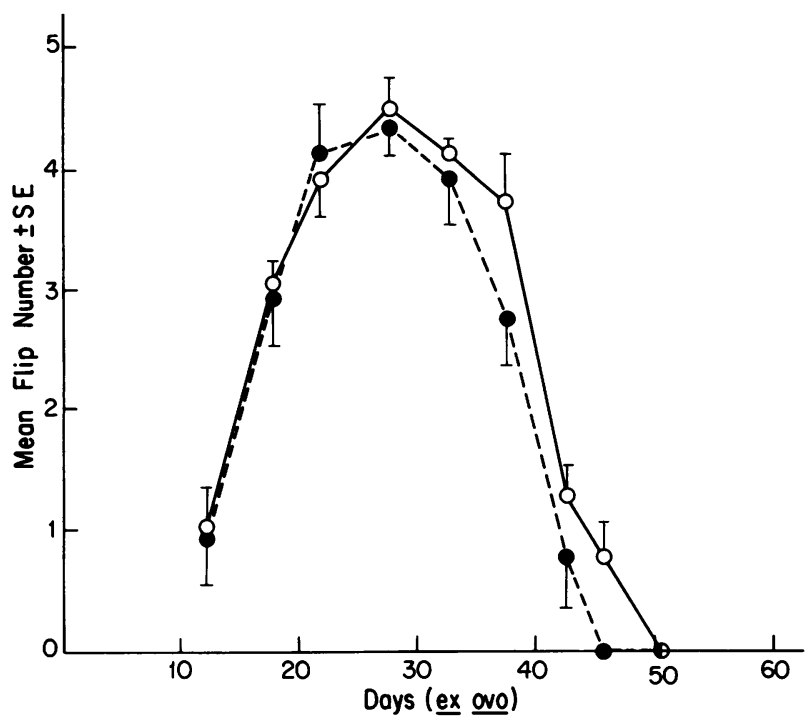

Figure 2 Effect of pepstatin on mean FN of Line 413 dystrophic chickens. Details of dosage schedule and procedure for flip testing are as described in Methods. The number of animals per point is 5 untreated dystrophic control $(\bullet)$ and 10 pepstatin-treated dystrophic (O).

3) compared with the corresponding untreated dystrophic control chickens. Normal control chickens reached a perfect mean $\mathrm{FN}$ of five at day 15 ex ovo and subsequently maintained this FN throughout the duration of the trials (data not shown).

Plasma creatine phosphokinase activity. As seen in Fig. 4, there were significantly $(P<0.001)$ higher

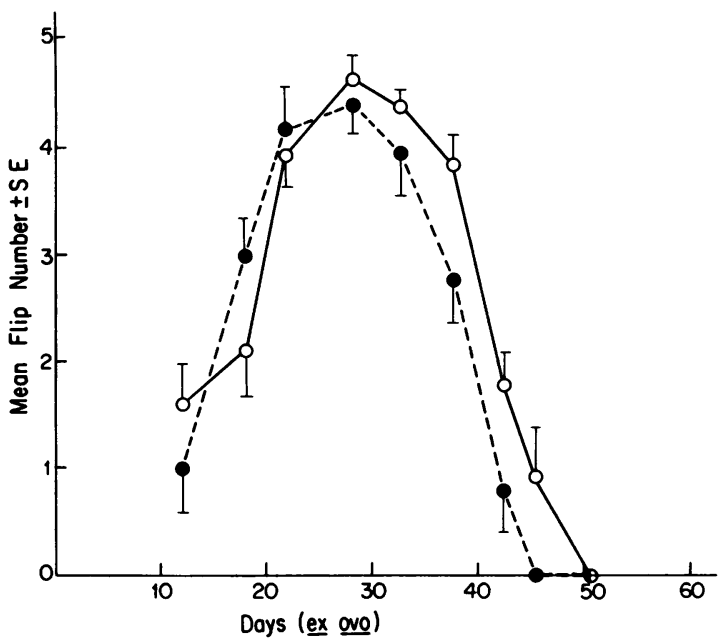

FIGURE 3 Effect of antipain on mean FN of Line 413 dystrophic chickens. Details of dosage schedule and procedure for flip testing are as described in Methods. The number of animals per point is five untreated dystrophic control $(\bullet)$ and seven antipain-treated dystrophic (O).

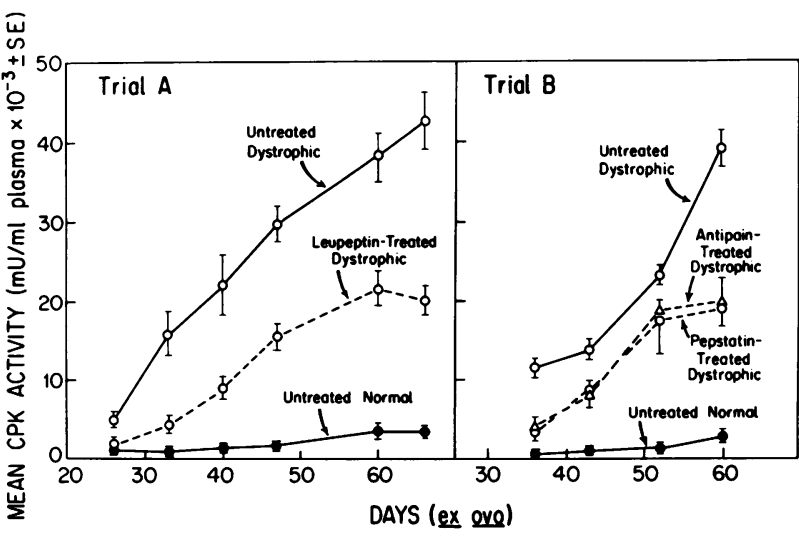

FIgURE 4 Plasma CPK activities from Line 413 dystrophic chickens treated with protease inhibitors. In all ages examined

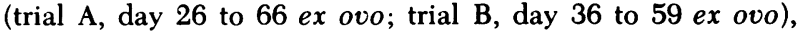
plasma CPK of the untreated dystrophic animals was significantly higher than the corresponding normal control group at $P<0.001$, and significantly greater than values from the leupeptin- (trial A), pepstatin-, and antipain-treated (trial B) dystrophic groups at $P<0.001$. CPK activity was determined as described in Methods. The numbers of identical animals bled on consecutive weeks are as follows: trial A, four untreated normal, four untreated dystrophic, and six leupeptin-treated dystrophic. Trial B, four untreated normal, three untreated dystrophic, four pepstatin-treated dystrophic, and four antipain-treated dystrophic.

levels of CPK activity in the plasma of dystrophic Line 413 chickens compared with the normally low values exhibited in the nondystrophic Line 412 chickens in each age group. Though CPK values were much higher than normal, significant reductions $(P<0.001)$ in plasma CPK were elicited in dystrophic chickens treated with leupeptin (Fig. 4, trial A), pepstatin, or antipain (Fig. 4, trial B). Each of these reductions was found throughout the duration of the drug trial.

Quantitation of muscle histology. In pectoralis major muscle from the untreated normal Line 412 chickens, no fiber necrosis, regeneration, vacuolar change, or inflammation was present (Table I). However, pathologic features observed in muscle from untreated dystrophic chickens were quite uniform. Vacuolar change, fiber necrosis, regenerative activity, and inflammatory cells were widespread in all specimens. Vacuolar change in the dystrophic myofiber occurred as multiple, round, clear, unstained sarcoplasm. Necrotic and degenerative fibers were granular and fragmented, and were often accompanied by phagocytic activity. Regenerating fibers, easily recognized in hematoxylin and eosin stains by their blue sarcoplasm (23), typically had multiple, large vesicular nuclei with prominent nucleoli. Inflammatory infiltrates found in the dystrophic muscle were primarily perivascular. They were composed of a monotonous, mononuclear 
TABLE I

Quantitation of Pectoralis Major Muscle Histology

\begin{tabular}{llccc}
\hline \multicolumn{1}{c}{ Experimental condition } & $n$ & $\begin{array}{c}\text { Degenerating and } \\
\text { regenerating fibers }\end{array}$ & $\begin{array}{c}\text { Vacuolated } \\
\text { fibers }\end{array}$ & $\begin{array}{c}\text { Inflammatory } \\
\text { cells }\end{array}$ \\
\hline Normal-untreated & 5 & None & None & None \\
Dystrophic-untreated & 7 & $4.7 \pm 0.7$ & $15.7 \pm 2.0$ & $7.3 \pm 2.1$ \\
Dystrophic-leupeptin & 6 & $4.8 \pm 0.5^{*}$ & $12.9 \pm 1.8^{*}$ & $4.5 \pm 0.8^{*}$ \\
Dystrophic-pepstatin & 6 & $3.1 \pm 0.7^{*}$ & $5.8 \pm 1.3 \ddagger$ & $4.6 \pm 1.3^{*}$ \\
Dystrophic-antipain & 5 & $3.7 \pm 0.7^{*}$ & $4.6 \pm 1.7 \ddagger$ & $3.8 \pm 1.9^{*}$ \\
\hline
\end{tabular}

For drug schedule and histologic procedures see Methods. Day 67 ex ovo birds were sacrificed at the completion of the drug trial. Values are expressed as the mean number of observations \pm SEM per square millimeter, and are based on the enumeration of sixteen separate $1-\mathrm{mm}^{2}$ grids per muscle sample.

* Difference from the corresponding untreated dystrophic group is not significant.

\$ Difference from the corresponding untreated dystrophic group is significant at $P<0.01$.

cellular population of lymphoid cells having small, dark nuclei and little cytoplasm.

No significant differences were found in the relative frequencies of regenerating and degenerating fibers and inflammatory cells between muscle samples from untreated dystrophic controls and chickens receiving protease inhibitor treatment (Table I). However, there were significant reductions $(P<0.01)$ in the mean number of vacuolated fibers found in a square millimeter of muscle from pepstatin- or antipain-treated chickens, compared with the untreated dystrophic controls (Table I).

Body and tissue weights. Little if any change in body weight was found in the mainly female dystrophic chickens treated with the various protease inhibitors compared with the corresponding untreated female dystrophic controls. Significant increases of 31.0, 30.5, and $14.8 \%$ were found in the wet weight of the afflicted pectoralis major muscle (expressed as percent body weight) from leupeptin-, pepstatin-, and antipaintreated dystrophic chickens, respectively. In this regard, there was no significant difference found in noncollagen protein of dystrophic muscle (mean 101.5 $\pm 22.0 \mathrm{mg}$ noncollagen protein/g wet wt muscle) compared with muscle from chickens treated with any of the protease inhibitors $(95.5 \pm 17.5 \mathrm{mg} / \mathrm{g})$. However, it should be noted that the muscle noncollagen protein from either the drug-treated or untreated dystrophic chickens was found to be significantly less $(P<0.001)$ than that measured in comparable normal chicken muscle $(227.3 \pm 17.2 \mathrm{mg} / \mathrm{g})$.

Compared with the untreated dystrophic controls, small increases $(4.0,12.1$, and $5.4 \%)$ in the liver wet weight were found in dystrophic chickens treated with leupeptin, pepstatin, and antipain, respectively. No change in the heart tissue was observed with any of the drug therapies.

\section{DISCUSSION}

In contrast to the significant enhancement in righting ability exhibited in Line 413 dystrophic chickens treated with either antiserotoninergic drugs $(20,21)$ or diphenylhydantoin (24-26), no remarkable improvement was demonstrated with protease inhibitor treatment. The temporal benefit in righting ability derived from leupeptin may be due in part to intraperitoneal leupeptin being able to penetrate muscle tissue better than either pepstatin or antipain $(11,27)$. However, it should be noted here that the FN remains a standardized measurement of functional ability or disability in the chicken and is not a direct quantitation of inherent muscle strength or weakness $(18-21,24,25)$. In future drug trials, a technique from Cabe et al. $(28,29)$ will be adapted to the chicken to determine the relative strength of the primary wing muscles. They use a sensitive strain gauge to assess directly the maximal strength of rat and mouse hindlimb and forelimb muscles in routine toxicological studies.

The abnormal elevation in blood CPK activity in dystrophic chickens (18-21, 24, 25, 30) and humans (31) is generally accepted as an indirect indicator of the onset and rate of progression of the muscle disease. In this connection, the significant reductions in plasma CPK levels found with each of the protease inhibitors (given intraperitoneally) can be perceived as therapeutically beneficial. This finding compares with previous reports of CPK reductions in dystrophic chickens given either subcutaneous (32) or intramuscular (17) injections of protease inhibitors. However, caution should be used in interpreting the relative value of reduced blood CPK as a criterion of drug-related benefit. For example, Munsat and Bradley (33) found that decreases in CPK levels were not accompanied by significant clinical improvement in individuals with facioscapulohumeral dystrophy treated with prednisone. 
The effect of each protease inhibitor on the dystrophic pectoralis major muscle histology is inconclusive. In the present study, no significant differences in the relative frequencies of degenerating and regenerating muscle fibers or of inflammatory cells were noted with treatment. In addition, the relative reduction in the mean number of vacuolated fibers is masked by the variability of these observations among individual muscle specimens, and between treated and untreated animals. To a great degree, these findings do not concur with the improved histological appearance previously reported for pectoralis muscle from dystrophic chickens treated with pepstatin and leupeptin (17), and for the anterior tibialis muscle from dystrophic mice treated with pepstatin (15). However, the drug-related increases in muscle mass and in noncollagen protein found here are very encouraging and are consistent with the increased myofiber areas observed microscopically in muscles from protease inhibitor-treated dystrophic chickens (17) and mice (15).

The major benefits from this trial of intraperitoneally administered leupeptin, pepstatin, or antipain are significant reductions in plasma CPK and net increases in pectoralis major muscle mass and noncollagen protein. It remains unresolved whether there is a drug-induced enhancement in actual muscle strength capability, which has been assessed indirectly by either the functional test for righting ability, quantitative muscle histology, or myofibrillar protein content (viz., noncollagen protein).

\section{ACKNOWLEDGMENTS}

The authors wish to thank Mr. James A. Stamos for his preparation of the illustrations, and Mr. Barry Wolitzky for his technical assistance.

Michael S. Hudecki holds a Research Career Development Award from the National Institute of Neurologic Disease and Stroke (NINDS). This work was supported by grants from the Muscular Dystrophy Association and NINDS.

\section{REFERENCES}

1. Kar, N. C., and C. M. Pearson. 1977. Hydrolytic enzymes and human muscular dystrophy. In Pathogenesis of $\mathrm{Hu}-$ man Muscular Dystrophies. L. P. Rowland, editor. Excerpta Medica, Amsterdam, The Netherlands. 387-394.

2. Kar, N. C., and C. M. Pearson. 1978. Muscular dystrophy and activation of proteinases. Muscle of Nerve. 1: 308-313.

3. Pennington, R. J., and J. E. Robinson. 1968. Cathepsin activity in normal and dystrophic human muscle. Enzymol. Biol. Clin. 9: 175-182.

4. Peterson, D. W., A. L. Lilyblade, and D. C. Bond. 1972. Proteolytic activity during growth of hypertrophic and atrophic muscles of genetically dystrophic chickens. Proc. Soc. Exp. Biol. Med. 141: 1056-1062.

5. Iodice, A. A., J. Chin, S. Perker, and I. M. Weinstock. 1972. Cathepsins A, B, C, D and autolysis during development of breast muscle of normal and dystrophic chickens. Arch. Biochem. Biophys. 152: 166-174.
6. Meyer, W. L., B. W. Little, J. R. Feussner, and D. H. Meyer. 1972. On the role of neutral hydrolases in muscle protein turnover. In Research in Muscle Development and the Muscle Spindle. B. Q. Banker, R. J. Przybylski, J. P. Van Der Meulen, and M. Victor, editors. Excerpta Medica, Amsterdam, The Netherlands. 195-217.

7. Pennington, R. J. 1963. Biochemistry of dystrophic muscle. 2. Some enzyme changes in dystrophic mouse muscle. Biochem. J. 88: 64-68.

8. Umezawa, H. 1972. Enzyme Inhibitors of Microbial Origin. University Park Press, Baltimore, Md.

9. Aoyagi, T., and H. Umezawa. 1975. Structures and activities of protease inhibitors of microbial origin. In Proteases and Biological Control. E. Reich. D. B. Rifkin, and E. Shaw, editors. Cold Spring Harbor Laboratory, Cold Spring Harbor, N. Y. 429-454.

10. Ionasescu, V., R. Ionasescu, D. Witte, R. Feld, P. Concilla, L. Karding, L. Kraus, and L. Stern. 1980. Altered protein synthesis and creatine kinase in breast muscle cell cultures from dystrophic chick embryos-corrective influence of leupeptin, pepstatin and diphenylhydantoin. $J$. Neurol. Sci. 46: 157-168.

11. Libby, P., and A. L. Goldberg. 1978. Leupeptin, a protease inhibitor, decreases protein degradation in normal and diseased muscles. Science (Wash. D. C.). 199: 534-536.

12. Iodice, A. A. 1976. The inhibition by pepstatin of cathepsin D and autolysis of dystrophic muscle. Life Sci. 19: $1351-1358$.

13. Sugita, H., S. Ishiura, K. Suzuki, and K. Imahori. 1980. Ca-activated neutral protease and its inhibitors: in vitro effect on intact myofibrils. Muscle \& Nerve. 3: 335-339.

14. McGowan, E. B., S. A. Shafiq, and A. Stracher. 1976. Delayed degeneration of dystrophic and normal muscle cell cultures treated with pepstatin, leupeptin, and antipain. Exp. Neurol. 50: 649-657.

15. Chelmicka-Schorr, E. E., B. G. W. Arnason, K. E. Astrom, and Z. Darzynkiewicz. 1978. Treatment of mouse muscular dystrophy with the protease inhibitor pepstatin.J. Neuropathol. Exp. Neurol. 37: 263-268.

16. Enomoto, A., and W. G. Bradley. 1977. Therapeutic trials in muscular dystrophy. III. Studies of microbial proteinase inhibitors in murine dystrophy. Arch. Neurol. 34: 771-773.

17. Stracher, A., E. B. McGowan, and S. A. Shafiq. 1978. Muscular dystrophy: inhibition of degeneration in vivo with protease inhibitors. Science (Wash. D. C.). 200: 50-51.

18. Hudecki, M. S., and E. A. Barnard. 1976. Retardation of symptoms of dystrophy in genetically dystrophic chickens by chemotherapy. Res. Commun. Chem. Pathol. Pharmacol. 14: 167-176.

19. Hudecki, M. S., C. Pollina, A. K. Bhargava, J. E. Fitzpatrick, C. A. Privitera, and D. Schmidt. 1978. Effect of exercise on chickens with hereditary muscular dystrophy. Exp. Neurol. 61: 65-73.

20. Hudecki, M. S., C. M. Pollina, A. K. Bhargava, R. S. Hudecki, and R. R. Heffner. 1979. Delayed functional disability in dystrophic chickens receiving chemotherapy. Muscle \& Nerve. 2: 57-67.

21. Hudecki, M. S., C. M. Pollina, A. K. Bhargava, and R. S Hudecki. 1980. Screening of anti-serotoninergic drugs employing the genetically dystrophic chicken. Arch. Neurol. 37: 545-550.

22. Lowry, P., N. J. Rosebrough, A. L. Farr, and R. J. Randall. 1951. Protein measurement with the Folin phenol reagent. J. Biol. Chem. 193: 265-275. 
23. Dubowitz, V., and M. H. Brooke. 1973. Muscle Biopsy: a modern approach. Saunders, London. 87.

24. Entrikin, R. K., K. L. Swanson, P. M. Weidoff, G. T. Patterson, and B. W. Wilson. 1977. Avian muscular dystrophy: functional and biochemical improvement with diphenylhydantoin. Science (Wash. D. C.). 195: 873-875.

25. Entrikin, R. K., G. T. Patterson, P. M. Weidoff, and B. W. Wilson. 1978. Righting ability and skeletal muscle properties of phenytoin-treated dystrophic chickens. Exp. Neurol. 61: 650-663.

26. Hudecki, M. S., C. M. Pollina, R. R. Heffner, and A. K. Bhargava. 1981. Enhanced functional ability in drugtreated dystrophic chickens: trial results with indomethacin, diphenylhydantoin, and prednisone. Exp. Neurol. In press.

27. Libby, P., J. S. Ingwall, and A. L. Goldberg. 1979. Reduction of protein degradation and atrophy in cultured fetal muscle hearts by leupeptin. Am. J. Physiol. 237: 35-39.

28. Cabe, P. A., H. A. Tilson, C. L. Mitchell, and R. Dennis.
1978. A simple recording grip strength device. Pharmacol. Biochem. Behav. 8: 101-102.

29. Cabe, P. A., and H. A. Tilson. 1978. Hind limb extensor response: a method for assessing motor dysfunction in rats. Pharmacol. Biochem. Behav. 9: 133-136.

30. Chou, T., E. J. Hill, E. Bartle, K. Wooley, V. LeQuire, W. Olson, R. Roeloffs, and J. H. Parks. 1975. Beneficial effects of penicillamine treatment on hereditary avian muscular dystrophy. J. Clin. Invest. 56: 842-849.

31. Munsat, T. L., R. Baloh, C. M. Pearson, and W. Fowler. 1973. Serum enzyme alterations in neuromuscular diseases. J. Am. Med. Assoc. 226: 1536-1543.

32. Sugita, H., T. Shimizu, S. Ishiura, T. Katamoto, and K. Suzuki. 1978. The progressive muscular dystrophy (PMD) and $\mathrm{Ca}^{++}$activated neutral protease (CANP). Fourth International Congress on Neuromuscular Diseases, Montreal, Canada. 151. (Abstr.)

33. Munsat, T. L., and W. G. Bradley. 1977. Serum creatine phosphokinase levels and prednisone-treated muscle weakness. Neurology. 27: 96-97. 